\title{
Probabilitas Osilasi Neutrino dan Kebergantungan Fase CP
}

\author{
Nurhadi* dan Agus Purwanto $^{\dagger}$ \\ Jurusan Fisika, Fakultas Matematika dan Ilmu Pengetahuan Alam, \\ Institut Teknologi Sepuluh Nopember (ITS), Kampus ITS Sukolilo, Surabaya 60111
}

\begin{abstract}
Intisari
Neutrino merupakan salah partikel elementer dari keluarga lepton. Ketika dipostulatkan oleh Pauli tahun 1930, neutrino diasumsikan tidak bermassa. Model standar dari fisika partikel juga memprediksi bahwa neutrino juga tidak bermassa. Tetapi, tahun 1998 percobaan di Super-Kamiokande berhasil mengamati osilasi neutrino di alam. Fenomena osilasi neutrino bisa dijelaskan hanya kalau neutrino bermassa. Sampai saat ini diketahui neutrino mempunyai tiga flavor yaitu neutrino elektron, neutrino muon, dan neutrino tauon. Keadaan eigen flavor dan keadaan eigen massa neutrino di hubungkan oleh matrik uniter MNS (Maki-Nakagawa-Sakata), yang mempunyai empat parameter yaitu tiga sudut dan satu fase CP. Dalam paper ini akan dikaji probabilitas osilasi neutrino tiga generasi baik dalam vakum maupun materi dan kebergantungan fase $\mathrm{CP}$.
\end{abstract}

\begin{abstract}
Neutrino is an elementary particle of lepton family. When postulated by Pauli in 1930, neutrinos were assumed massless. The standard model of particle physics predicts that neutrinos are also massless. But, in 1998, SuperKamiokande experiment successfully observe neutrino oscillations in nature. The phenomenon of neutrino oscillations can be explained only if neutrinos have mass. Currently there are three known neutrino flavor i.e electron neutrinos, mu neutrinos, and tau neutrinos. Eigen state of flavor and eigen state of neutrino masses connected by a unitary matrix MNS (Maki-Nakagawa-Sakata), who has four parameters, namely three angles and the one phase CP. In this paper examined three generations of neutrino oscillation probability in both the vacuum and the matter and the dependence of the phase $\mathrm{CP}$.
\end{abstract}

KATA KUNCI: neutrino oscillation, vacuum, matter

\section{PENDAHULUAN}

Neutrino merupakan salah satu partikel elementer dari keluarga lepton (yang lain kuark) yang dipercaya sebagai penyusun alam semesta ini. Pertama kali neutrino diusulkan oleh Pauli pada tahun 1930 untuk menjelaskan fenomena peluruhan beta dan tidak bermassa. Pada tahun 1956, Cowan dan Reines menemukan anti neutrino. Neutrino merupakan partikel yang unik karena tidak bermuatan dan hanya berinteraksi lemah saja. Sampai saat ini neutrino diketahui memiliki tiga rasa (flavor) yaitu neutrino elektron, neutrino muon dan neutrino tauon. Selanjutnya model standar fisika partikel juga memprediksi kalau neutrino tidak bermassa, tetapi tahun 1998 percobaan Super-Kamiokande memberikan bukti kuat mengenai kehadiran fenomena osilasi neutrino yang berimplikasi neutrino bermassa [1]. Selain itu adanya persoalan neutrino matahari yaitu terjadinya perbedaan fluks neutrino elektron yang diterima ketika sampai di bumi dengan prediksi teoritis. Fluks neutrino elektron yang diterima oleh percobaan ternyata cuma sepertiga dari prediksi teoretis. Persoalan ini bisa diselesaikan dengan osilasi neutrino dan mempunyai massa walaupun kecil.

\footnotetext{
*E-MAIL: nurhadi10@mhs.physics.its.ac.id
}

†E-MAIL: purwanto@physics.its.ac.id
Jauh sebelumnya osilasi neutrino sebenarnya telah diprediksi oleh Maki, et al. [2] pada tahun 1962. Maki menunjukkan osilasi neutrino dua generasi yaitu neutrino elektron ke neutrino muon dan memprediksi bahwa neutrino bermassa walaupun sangat kecil. Sekarang diketahui neutrino mempunyai tiga generasi yaitu neutrino elektron, neutrino muon, dan neutrino tauon. Karena tiga generasi tersebut, maka matrik MNS nya menjadi $3 \times 3$ yang mempunyai empat parameter yaitu tiga sudut $\left(\theta_{12}, \theta_{23}, \theta_{13}\right)$ dan satu sudut fase CP. Pada penelitian sebelumnya [3-5] menyajikan bagaimana mendapatkan fase CP baik secara teoritis maupun percobaan. Pada tahun 2014 ketiga parameter sudut pada matrik MNS sudah berhasil dikonfirmasi oleh percobaan, tetapi fase $\mathrm{CP}$ masih menjadi tantangan tersendiri untuk dikonfirmasi oleh percobaan.

\section{PROBABILITAS OSILASI NEUTRINO DAN KEBERGANTUNGAN FASE CP DALAM VAKUM}

Untuk mendapatkan perumusan probabilitas osilasi neutrino dalam vakum, tinjau Hamiltonian neutrino tiga generasi

$$
H=\left(\begin{array}{ccc}
H_{e e} & H_{e \mu} & H_{e \tau} \\
H_{\mu e} & H_{\mu \mu} & H_{\mu \tau} \\
H_{\tau e} & H_{\tau \mu} & H_{\tau \tau}
\end{array}\right)
$$


diagonalisasi Hamiltonian memberikan

$$
U^{\dagger} H U=\left(\begin{array}{ccc}
E_{1} & & \\
& E_{2} & \\
& & E_{2}
\end{array}\right)
$$

dengan $E_{i}=E+\frac{m_{i}^{2}}{2 E}$, maka

$$
\begin{aligned}
U^{\dagger} H U & =\left(\begin{array}{ccc}
E+\frac{m_{1}^{2}}{2 E} & & \\
& E+\frac{m_{2}^{2}}{2 E} & \\
& & E+\frac{m_{3}^{2}}{2 E}
\end{array}\right) \\
& =E+\frac{1}{2 E}\left(\begin{array}{lll}
m_{1}^{2} & & \\
& m_{2}^{2} & \\
& & m_{3}^{2}
\end{array}\right)
\end{aligned}
$$

keadaan eigen dari neutrino diberikan

$$
\left|\nu_{\alpha}\right\rangle=\sum_{i} U_{\alpha i}^{*}\left|\nu_{i}\right\rangle
$$

dengan $U_{\alpha i}$ adalah matrik bauran neutrino dan keadaan eigen neutrino yang bergantung waktu diberikan

$$
\left|\nu_{\alpha}(t)\right\rangle=\sum_{i} U_{\alpha i}^{*} e^{-i E_{i} t}\left|\nu_{i}\right\rangle
$$

dan

$$
\left\langle\nu_{\beta}\right|=\sum_{i}\left\langle\nu_{\beta}\right| U_{\beta i}
$$

sehingga amplitudo transisi neutrino

$$
\begin{aligned}
\left\langle\nu_{\beta} \mid \nu_{\alpha}(t)\right\rangle & =\sum_{j} \sum_{i} U_{\alpha i}^{*} e^{-i E_{i} t} U_{\beta j}\left\langle\nu_{j} \mid \nu_{i}\right\rangle \\
& =\sum_{i} U_{\alpha i}^{*} e^{-i E_{i} t} U_{\beta i} \\
& =\sum_{i} U_{\alpha i}^{*} e^{-i\left(E+\frac{m_{i}^{2}}{2 E}\right) t} U_{\beta i} \\
& =e^{-i E t} \sum_{i} U_{\alpha i}^{*} e^{-i \frac{m_{i}^{2}}{2 E} t} U_{\beta i} \\
& =e^{-i E L} \sum_{i} U_{\alpha i}^{*} e^{-i \frac{m_{i}^{2}}{2 E} L} U_{\beta i}
\end{aligned}
$$

dan amplitudo probabilitasnya diberikan

$$
\begin{aligned}
P\left(\nu_{\alpha} \rightarrow \nu_{\beta}\right) & =\left|A\left(\nu_{\alpha} \rightarrow \nu_{\beta}\right)\right|^{2} \\
& =\delta_{\alpha \beta}-4 \sum_{(i j)}^{\text {siklik }} R e J_{\alpha \beta}^{i j} \sin ^{2} \Delta_{i j}^{\prime} \\
& -2 \sum_{(i j)}^{s i k l i k} I m J_{\alpha \beta}^{i j} \sin 2 \Delta_{i j}^{\prime}
\end{aligned}
$$

dengan

$$
\begin{aligned}
J_{\alpha \beta}^{i j} & =U_{\alpha i} U_{\beta i}^{*} U_{\alpha j}^{*} U_{\beta j} \\
\Delta_{i j}^{\prime} & \equiv \frac{\Delta_{i j} L}{4 E} \equiv \frac{\left(m_{i}^{2}-m_{j}^{2}\right) L}{4 E}
\end{aligned}
$$

Dengan menggunakan faktor Jarlskog (Jarlskog invarint), suku ketiga Pers.(8) dapat dituliskan

$$
\begin{aligned}
P\left(\nu_{\alpha} \rightarrow \nu_{\beta}\right) & =\delta_{\alpha \beta}-4 \sum_{(i j)}^{s i k l i k} \operatorname{Re} J_{\alpha \beta}^{i j} \sin ^{2} \Delta_{i j}^{\prime} \\
& \pm 2 \sum_{(i j)}^{\text {siklik }} J \sin 2 \Delta_{i j}^{\prime}
\end{aligned}
$$

Tanda \pm pada suku ketiga Pers. $(11)$, diambil $-(+)$ dalam kasus $(\alpha, \beta)$ yang merupakan permutasi (anti) siklik dari $(e, \mu)$. Penjumlahan siklik terhadap seluruh $(i j)=(12),(23),(31)$. Selanjutnya akan di hitung secara eksplisit $R e J_{\alpha \beta}^{i j}=U_{\alpha i} U_{\beta i}^{*} U_{\alpha j}^{*} U_{\beta j}$ dan faktor Jarlskog $J=\operatorname{Im}\left(U_{e 1} U_{\mu 1}^{*} U_{e 2}^{*} U_{\mu 2}\right)$. Dengan menggunakan matriks bauran untuk neutrino (matriks MNS) mempunyai bentuk

$$
U_{\alpha i}=\left(\begin{array}{ccc}
c_{12} c_{13} & s_{12} c_{13} & s_{13} e^{-i \delta} \\
-s_{12} c_{23}-c_{12} s_{23} s_{13} e^{i \delta} & c_{12} c_{23}-s_{12} s_{23} s_{13} e^{i \delta} & s_{23} c_{13} \\
s_{12} s_{23}-c_{12} c_{23} s_{13} e^{i \delta} & -c_{12} s_{23}-s_{12} c_{23} s_{13} e^{i \delta} & c_{23} c_{13}
\end{array}\right)
$$


didapatkan bentuk eksplisit

$$
\begin{aligned}
\operatorname{Re} J_{e \mu}^{12}= & -\left(c_{12}^{2}-s_{12}^{2}\right) J_{r} \cos \delta+s_{12}^{2} c_{12}^{2} c_{13}^{2}\left(s_{23}^{2} s_{13}^{2}-c_{23}^{2}\right) \\
\operatorname{Re} J_{e \mu}^{23}= & J_{r} \cos \delta-s_{12}^{2} s_{23}^{2} s_{13}^{2} c_{13}^{2} \\
\operatorname{Re} J_{e \mu}^{31}= & -J_{r} \cos \delta-c_{12}^{2} s_{23}^{2} s_{13}^{2} c_{13}^{2} \\
\operatorname{Re} J_{e \tau}^{12}= & \left(c_{12}^{2}-s_{12}^{2}\right) J_{r} \cos \delta+s_{12}^{2} c_{12}^{2} c_{13}^{2}\left(c_{23}^{2} s_{13}^{2}-s_{23}^{2}\right) \\
\operatorname{Re} J_{e \mu}^{23}= & -J_{r} \cos \delta-s_{12}^{2} c_{23}^{2} s_{13}^{2} c_{13}^{2} \\
\operatorname{Re} J_{e \tau}^{31}= & J_{r} \cos \delta-c_{12}^{2} c_{23}^{2} s_{13}^{2} c_{13}^{2} \\
\operatorname{Re} J_{\mu \tau}^{12}= & s_{12}^{2} c_{12}^{2} s_{23}^{2} c_{23}^{2}\left(1+s_{13}^{2}+s_{13}^{4}\right)-\left(s_{12}^{2} c_{12}^{2}+s_{23}^{2} c_{23}^{2}\right) s_{13}^{2} \\
& -\left(c_{12}^{2}-s_{12}^{2}\right)\left(c_{23}^{2}-s_{23}^{2}\right) s_{12} c_{12} s_{23} c_{23} s_{13}\left(1+s_{13}^{2}\right) \cos \delta+2 s_{12}^{2} c_{12}^{2} s_{23}^{2} c_{23}^{2} s_{13}^{2} \cos 2 \delta \\
\operatorname{Re} J_{\mu \tau}^{23}= & -\left(c_{23}^{2}-s_{23}^{2}\right) J_{r} \cos \delta+s_{23}^{2} c_{23}^{2} c_{13}^{2}\left(c_{12}^{2} s_{13}^{2}-s_{12}^{2}\right) \\
\operatorname{Re} J_{\mu \tau}^{31}= & \left(c_{23}^{2}-s_{23}^{2}\right) J_{r} \cos \delta+s_{23}^{2} c_{23}^{2} c_{13}^{2}\left(c_{12}^{2} s_{13}^{2}-s_{12}^{2}\right)
\end{aligned}
$$

dengan $s_{i j} \equiv \sin _{i j}, c_{i j} \equiv \cos _{i j}$ dan faktor Jarlskog

$$
J=s_{12} c_{12} s_{23} c_{23} s_{13} c_{13}^{2} \sin \delta=J_{r} \sin \delta
$$

Probabilitas osilasi neutrino baik transisi maupun survival dalam vakum didapatkan dengan memasukkan bentuk eksplisit $R e J_{\alpha \beta}^{i j}$ dan faktor Jarlskog ke Pers.(11), didapatkan

$$
\begin{aligned}
& P\left(\nu_{e} \rightarrow \nu_{\mu}\right)=A_{e \mu} \cos \delta+B \sin \delta+C_{e \mu} \\
& P\left(\nu_{e} \rightarrow \nu_{\tau}\right)=-A_{e \mu} \cos \delta-B \sin \delta+C_{e \tau} \\
& P\left(\nu_{\mu} \rightarrow \nu_{\tau}\right)=A_{\mu \tau} \cos \delta+B \sin \delta+C_{\mu \tau}+D \cos 2 \delta \\
& P\left(\nu_{e} \rightarrow \nu_{e}\right)=C_{e e} \\
& P\left(\nu_{\mu} \rightarrow \nu_{\mu}\right)=A_{\mu \mu} \cos \delta+C_{\mu \mu}-D \cos 2 \delta \\
& P\left(\nu_{\tau} \rightarrow \nu_{\tau}\right)=A_{\tau \tau} \cos \delta+C_{\tau \tau}-D \cos 2 \delta
\end{aligned}
$$

dengan koefisien-koefisiennya secara eksplisit adalah

$$
\begin{aligned}
A_{e \mu}= & \left\{4\left(c_{12}^{2}-s_{12}^{2}\right) J_{r} \sin ^{2}\left(\frac{\Delta_{12}}{4 E} L\right)-4 J_{r} \sin ^{2}\left(\frac{\Delta_{23}}{4 E} L\right)+4 J_{r} \sin ^{2}\left(\frac{\Delta_{31}}{4 E} L\right)\right\} \\
B= & \left\{-2 J_{r}\left(\sin \left(\frac{\Delta_{12}}{2 E} L\right)+\sin \left(\frac{\Delta_{23}}{2 E} L\right)+\sin \left(\frac{\Delta_{31}}{2 E} L\right)\right)\right\} \\
C_{e \mu}= & \left\{-4 s_{12}^{2} c_{12}^{2} c_{13}^{2}\left(s_{13}^{2} s_{23}^{2}-c_{23}^{2}\right) \sin ^{2}\left(\frac{\Delta_{12}}{4 E} L\right)+4 s_{12}^{2} s_{13}^{2} s_{23}^{2} c_{13}^{2} \sin ^{2}\left(\frac{\Delta_{23}}{4 E} L\right)\right. \\
& \left.+4 c_{12}^{2} s_{13}^{2} c_{13}^{2} s_{23}^{2} \sin ^{2}\left(\frac{\Delta_{31}}{4 E} L\right)\right\}
\end{aligned}
$$




$$
\begin{aligned}
& C_{\text {e } \tau}=\left\{-4 s_{12}^{2} c_{12}^{2} c_{13}^{2}\left(s_{13}^{2} s_{23}^{2}-c_{23}^{2}\right) \sin ^{2}\left(\frac{\Delta_{12}}{4 E} L\right)+4 s_{12}^{2} s_{13}^{2} s_{23}^{2} c_{13}^{2} \sin ^{2}\left(\frac{\Delta_{23}}{4 E} L\right)\right. \\
& \left.+4 c_{12}^{2} s_{13}^{2} c_{13}^{2} s_{23}^{2} \sin ^{2}\left(\frac{\Delta_{31}}{4 E} L\right)\right\} \\
& A_{\mu \tau}=\left\{+4\left(c_{12}^{2}-s_{12}^{2}\right)\left(c_{23}^{2}-s_{23}^{2}\right) s_{12} c_{12} s_{23} c_{23} s_{13}\left(1+s_{13}^{2}\right) \sin ^{2}\left(\frac{\Delta_{12}}{4 E} L\right)\right. \\
& \left.+4\left(c_{23}^{2}-s_{23}^{2}\right) J_{r} \sin ^{2}\left(\frac{\Delta_{23}}{4 E} L\right)-4\left(c_{23}^{2}-s_{23}^{2}\right) J_{r} \sin ^{2}\left(\frac{\Delta_{31}}{4 E} L\right)\right\} \\
& C_{\mu \tau}=\left\{-4 s_{12}^{2} c_{12}^{2} s_{23}^{2} c_{23}^{2}\left(1+s_{13}^{2}+s_{13}^{4}\right) \sin ^{2}\left(\frac{\Delta_{12}}{4 E} L\right)+4\left(s_{12}^{2} c_{12}^{2}+s_{23}^{2} c_{23}^{2}\right) s_{13}^{2} \sin ^{2}\left(\frac{\Delta_{12}}{4 E} L\right)\right. \\
& \left.-4 s_{23}^{2} c_{23}^{2} c_{13}^{2}\left(c_{12}^{2} s_{13}^{2}-s_{12}^{2}\right) \sin ^{2}\left(\frac{\Delta_{31}}{4 E} L\right)-4 s_{23}^{2} c_{23}^{2} c_{13}^{2}\left(c_{12}^{2} s_{13}^{2}-s_{12}^{2}\right) \sin ^{2}\left(\frac{\Delta_{31}}{4 E} L\right)\right\} \\
& D=\left\{-8 s_{12}^{2} c_{12}^{2} s_{23}^{2} c_{23}^{2} s_{13}^{2} \sin ^{2}\left(\frac{\Delta_{12}}{4 E} L\right)\right\} \\
& C_{e e}=1-C_{e \mu}-C_{e \tau} \\
& =1-\left\{-4 s_{12}^{2} c_{12}^{2} c_{13}^{2}\left(s_{13}^{2} s_{23}^{2}-c_{23}^{2}\right) \sin ^{2}\left(\frac{\Delta_{12}}{4 E} L\right)\right. \\
& +4 s_{12}^{2} s_{13}^{2} s_{23}^{2} c_{13}^{2} \sin ^{2}\left(\frac{\Delta_{23}}{4 E} L\right) \\
& \left.+4 c_{12}^{2} s_{13}^{2} c_{13}^{2} s_{23}^{2} \sin ^{2}\left(\frac{\Delta_{31}}{4 E} L\right)\right\} \\
& -\left\{-4 s_{12}^{2} c_{12}^{2} c_{13}^{2}\left(s_{13}^{2} s_{23}^{2}-c_{23}^{2}\right) \sin ^{2}\left(\frac{\Delta_{12}}{4 E} L\right)\right. \\
& +4 s_{12}^{2} s_{13}^{2} s_{23}^{2} c_{13}^{2} \sin ^{2}\left(\frac{\Delta_{23}}{4 E} L\right) \\
& \left.+4 c_{12}^{2} s_{13}^{2} c_{13}^{2} s_{23}^{2} \sin ^{2}\left(\frac{\Delta_{31}}{4 E} L\right)\right\} \\
& A_{\mu \mu}=-A_{e \mu}-A_{\mu \tau} \\
& =-\left\{4\left(c_{12}^{2}-s_{12}^{2}\right) J_{r} \sin ^{2}\left(\frac{\Delta_{12}}{4 E} L\right)-4 J_{r} \sin ^{2}\left(\frac{\Delta_{23}}{4 E} L\right)\right. \\
& \left.+4 J_{r} \sin ^{2}\left(\frac{\Delta_{31}}{4 E} L\right)\right\} \\
& -\left\{+4\left(c_{12}^{2}-s_{12}^{2}\right)\left(c_{23}^{2}-s_{23}^{2}\right) s_{12} c_{12} s_{23} c_{23} s_{13}\left(1+s_{13}^{2}\right) \sin ^{2}\left(\frac{\Delta_{12}}{4 E} L\right)\right. \\
& +4\left(c_{23}^{2}-s_{23}^{2}\right) J_{r} \sin ^{2}\left(\frac{\Delta_{23}}{4 E} L\right) \\
& \left.-4\left(c_{23}^{2}-s_{23}^{2}\right) J_{r} \sin ^{2}\left(\frac{\Delta_{31}}{4 E} L\right)\right\}
\end{aligned}
$$




$$
\begin{aligned}
& C_{\mu \mu}=1-C_{e \mu}-C_{\mu \tau} \\
& =1-\left\{-4 s_{12}^{2} c_{12}^{2} c_{13}^{2}\left(s_{13}^{2} s_{23}^{2}-c_{23}^{2}\right) \sin ^{2}\left(\frac{\Delta_{12}}{4 E} L\right)\right. \\
& +4 s_{12}^{2} s_{13}^{2} s_{23}^{2} c_{13}^{2} \sin ^{2}\left(\frac{\Delta_{23}}{4 E} L\right) \\
& \left.+4 c_{12}^{2} s_{13}^{2} c_{13}^{2} s_{23}^{2} \sin ^{2}\left(\frac{\Delta_{31}}{4 E} L\right)\right\} \\
& -\left\{-4 s_{12}^{2} c_{12}^{2} s_{23}^{2} c_{23}^{2}\left(1+s_{13}^{2}+s_{13}^{4}\right) \sin ^{2}\left(\frac{\Delta_{12}}{4 E} L\right)\right. \\
& +4\left(s_{12}^{2} c_{12}^{2}+s_{23}^{2} c_{23}^{2}\right) s_{13}^{2} \sin ^{2}\left(\frac{\Delta_{12}}{4 E} L\right) \\
& -4 s_{23}^{2} c_{23}^{2} c_{13}^{2}\left(c_{12}^{2} s_{13}^{2}-s_{12}^{2}\right) \sin ^{2}\left(\frac{\Delta_{31}}{4 E} L\right) \\
& \left.-4 s_{23}^{2} c_{23}^{2} c_{13}^{2}\left(c_{12}^{2} s_{13}^{2}-s_{12}^{2}\right) \sin ^{2}\left(\frac{\Delta_{31}}{4 E} L\right)\right\} \\
& A_{\tau \tau}=A_{e \mu}-A_{\mu \tau} \\
& =\left\{4\left(c_{12}^{2}-s_{12}^{2}\right) J_{r} \sin ^{2}\left(\frac{\Delta_{12}}{4 E} L\right)-4 J_{r} \sin ^{2}\left(\frac{\Delta_{23}}{4 E} L\right)\right. \\
& \left.+4 J_{r} \sin ^{2}\left(\frac{\Delta_{31}}{4 E} L\right)\right\} \\
& -\left\{+4\left(c_{12}^{2}-s_{12}^{2}\right)\left(c_{23}^{2}-s_{23}^{2}\right) s_{12} c_{12} s_{23} c_{23} s_{13}\left(1+s_{13}^{2}\right) \sin ^{2}\left(\frac{\Delta_{12}}{4 E} L\right)\right. \\
& +4\left(c_{23}^{2}-s_{23}^{2}\right) J_{r} \sin ^{2}\left(\frac{\Delta_{23}}{4 E} L\right) \\
& \left.-4\left(c_{23}^{2}-s_{23}^{2}\right) J_{r} \sin ^{2}\left(\frac{\Delta_{31}}{4 E} L\right)\right\} \\
& C_{\mu \mu}=1-C_{e \mu}-C_{\mu \tau} \\
& =1-\left\{-4 s_{12}^{2} c_{12}^{2} c_{13}^{2}\left(s_{13}^{2} s_{23}^{2}-c_{23}^{2}\right) \sin ^{2}\left(\frac{\Delta_{12}}{4 E} L\right)\right. \\
& +4 s_{12}^{2} s_{13}^{2} s_{23}^{2} c_{13}^{2} \sin ^{2}\left(\frac{\Delta_{23}}{4 E} L\right) \\
& \left.+4 c_{12}^{2} s_{13}^{2} c_{13}^{2} s_{23}^{2} \sin ^{2}\left(\frac{\Delta_{31}}{4 E} L\right)\right\} \\
& -\left\{-4 s_{12}^{2} c_{12}^{2} s_{23}^{2} c_{23}^{2}\left(1+s_{13}^{2}+s_{13}^{4}\right) \sin ^{2}\left(\frac{\Delta_{12}}{4 E} L\right)\right. \\
& +4\left(s_{12}^{2} c_{12}^{2}+s_{23}^{2} c_{23}^{2}\right) s_{13}^{2} \sin ^{2}\left(\frac{\Delta_{12}}{4 E} L\right) \\
& -4 s_{23}^{2} c_{23}^{2} c_{13}^{2}\left(c_{12}^{2} s_{13}^{2}-s_{12}^{2}\right) \sin ^{2}\left(\frac{\Delta_{31}}{4 E} L\right) \\
& \left.-4 s_{23}^{2} c_{23}^{2} c_{13}^{2}\left(c_{12}^{2} s_{13}^{2}-s_{12}^{2}\right) \sin ^{2}\left(\frac{\Delta_{31}}{4 E} L\right)\right\}
\end{aligned}
$$

\section{PROBABILITAS OSILASI NEUTRINO DAN KEBERGANTUNGAN FASE CP DALAM MATERI}

Untuk mendapatkan perumusan probabilitas osilasi neutrino dalam materi, perhatikan hamiltonian neutrino tiga gen- erasi dalam materi berikut ini

$$
\begin{aligned}
\tilde{H} & =\left(\begin{array}{ccc}
\tilde{H}_{e e} & \tilde{H}_{e \mu} & \tilde{H}_{e \tau} \\
\tilde{H}_{\mu e} & \tilde{H}_{\mu \mu} & \tilde{H}_{\mu \tau} \\
\tilde{H}_{\tau e} & \tilde{H}_{\tau \mu} & \tilde{H}_{\tau \tau}
\end{array}\right) \\
& =H+\frac{1}{2 E}\left(\begin{array}{ccc}
A & & \\
& 0 & \\
& & 0
\end{array}\right)
\end{aligned}
$$


dengan $A=2 \sqrt{2} G_{F} \rho_{e} E$ adalah potensial dalam materi.

Diagonalisasi Hamiltonian dalam ini memberikan

$$
\begin{aligned}
\tilde{U}^{\dagger} \tilde{H} \tilde{U} & =\left(\begin{array}{ccc}
E_{1} & & \\
& E_{1} & \\
& & E_{2}
\end{array}\right) \\
& =\left(\begin{array}{ccc}
E+\frac{m_{1}^{2}}{2 E} & & \\
& E+\frac{m_{2}^{2}}{2 E} & \\
& & \\
& & E+\frac{m_{3}^{2}}{2 E}
\end{array}\right) \\
& =E+\frac{1}{2 E}\left(\begin{array}{lll}
m_{1}^{2} & & \\
& m_{2}^{2} & \\
& & m_{3}^{2}
\end{array}\right) \\
& =E+\frac{1}{2 E}\left(\begin{array}{lll}
\lambda_{1} & & \\
& \lambda_{2} & \\
& & \lambda_{3}
\end{array}\right)
\end{aligned}
$$

dengan $\lambda_{i}$ adalah nilai eigen massa dalam materi. Keadaan eigen neutrino dalama materi diberikan

$$
\left|\nu_{\alpha}\right\rangle=\sum_{i} \tilde{U}_{\alpha i}^{*}\left|\nu_{i}\right\rangle
$$

dengan $\tilde{U}_{\alpha i}$ adalah matriks bauran neutrino dalam materi dan keadaan eigen neutrino dalam materi yang bergantung waktu diberikan

$$
\left|\nu_{\alpha}(t)\right\rangle=\sum_{i} \tilde{U}_{\alpha i}^{*} e^{-i E_{i} t}\left|\nu_{i}\right\rangle
$$

dan

$$
\left\langle\nu_{\beta}\right|=\sum_{i}\left\langle\nu_{\beta}\right| \tilde{U}_{\beta i}
$$

sehingga amplitudo transisi neutrino dalam materi adalah

$$
\begin{aligned}
\left\langle\nu_{\beta} \mid \nu_{\beta}(t)\right\rangle & =\sum_{j} \sum_{i} \tilde{U}_{\alpha i}^{*} e^{-i E_{i} t} \tilde{U}_{\beta_{j}}\left\langle\nu_{j} \mid \nu_{i}\right\rangle \\
& =\sum_{i} \tilde{U}_{\alpha i}^{*} e^{-i E_{i} t} \tilde{U}_{\beta i} \\
& =\sum_{i} \tilde{U}_{\alpha i}^{*} e^{-i\left(E+\frac{m_{i}^{2}}{2 E}\right) t} \tilde{U}_{\beta i} \\
& =e^{-i E t} \sum_{i} \tilde{U}_{\alpha i}^{*} e^{-i \frac{\lambda_{i}}{2 E} t} \tilde{U}_{\beta i} \\
& =e^{-i E L} \sum_{i} \tilde{U}_{\alpha i}^{*} e^{-i \frac{\lambda_{i}}{2 E} L} \tilde{U}_{\beta i}
\end{aligned}
$$

Sehingga probabilitas osilasi neutrino dalam materi adalah seperti pada Pers.(11) adalah

$$
\begin{aligned}
P\left(\nu_{\alpha} \rightarrow \nu_{\beta}\right) & =\delta_{\alpha \beta}-4 \sum_{(i j)}^{\text {siklik }} \operatorname{Re} \tilde{J}_{\alpha \beta}^{i j} \sin ^{2} \tilde{\Delta}_{i j}^{\prime} \\
& \pm 2 \sum_{(i j)}^{s i k l i k} \tilde{J} \sin 2 \tilde{\Delta}_{i j}
\end{aligned}
$$

Untuk mendapatkan bentuk eksplisit probabilitas osilasi neutrino dalam materi, diperkenalkan $\tilde{p}_{\alpha \beta}$ dan $\tilde{q}_{\alpha \beta}$ yang didefi- nisikan

$$
\begin{aligned}
\tilde{p}_{\alpha \beta} & =2 E \tilde{H}_{\alpha \beta} \\
\tilde{q}_{\alpha \beta} & =(2 E)^{2} \tilde{\mathcal{H}}_{\alpha \beta} \\
& =(2 E)^{2}\left(\tilde{H}_{\gamma \beta} \tilde{H}_{\alpha \gamma}-\tilde{H}_{\alpha \beta} \tilde{H}_{\gamma \gamma}\right)
\end{aligned}
$$

dengan $(\alpha \beta \gamma)=(e \mu \tau),(\mu \tau e),(\tau e \mu)$. Dari sifat unitaritas matriks MNS dan Pers.(48) dan (49) didapatkan

1. Hubungan pertama unitaritas matriks MNS

$$
\sum_{i} \tilde{U}_{\alpha i} \tilde{U}_{\beta i}^{*}=\delta_{\alpha \beta}
$$

2. Hubungan kedua $\tilde{H}=\tilde{U} \operatorname{diag}\left(\lambda_{1}, \lambda_{2}, \lambda_{3}\right) \tilde{U}^{\dagger}$, didapatkan

$$
\sum_{i} \lambda_{i} \tilde{U}_{\alpha i} \tilde{U}_{\beta i}^{*}=\tilde{p}_{\alpha \beta}
$$

3. Hubungan ketiga

$$
\begin{aligned}
(2 E)^{2} \mathcal{H} & =(2 E)^{2} \tilde{H}^{-1}(\operatorname{det} \tilde{H}) \\
& =\tilde{U} \operatorname{diag}\left(\frac{1}{\lambda_{1}}, \frac{1}{\lambda_{2}}, \frac{1}{\lambda_{3}}\right) \tilde{U}^{\dagger} \times \lambda_{1} \lambda_{2} \lambda_{3}
\end{aligned}
$$

didapatkan

$$
\sum_{i j k} \lambda_{j} \lambda_{k} \tilde{U}_{\alpha i} \tilde{U}_{\beta i}^{*}=\tilde{q}_{\alpha \beta}
$$

dari ketiga hubungan tersebut didapatkan

$$
\begin{aligned}
\tilde{U}_{\alpha i} \tilde{U}_{\beta i}^{*} & =\frac{\tilde{p}_{\alpha \beta} \lambda_{i}+\tilde{q}_{\alpha \beta}-\delta_{\alpha \beta} \lambda_{i}\left(\lambda_{j}+\lambda_{k}\right)}{\tilde{\Delta}_{j i} \tilde{\Delta}_{k i}} \\
\tilde{U}_{\alpha j}^{*} \tilde{U}_{\beta j} & =\frac{\tilde{p}_{\alpha \beta}^{*} \lambda_{j}+\tilde{q}_{\alpha \beta}^{*}-\delta_{\alpha \beta} \lambda_{j}\left(\lambda_{k}+\lambda_{i}\right)}{\tilde{\Delta}_{i j} \tilde{\Delta}_{k j}}
\end{aligned}
$$

sehingga Pers.(47) dapat ditulis sebagai berikut

$$
\begin{aligned}
P\left(\nu_{\alpha} \rightarrow \nu_{\beta}\right) & =\delta_{\alpha \beta}-4 \sum_{(i j)}^{s i k l i k} \operatorname{Re} \tilde{J}_{\alpha \beta}^{i j} \sin ^{2} \tilde{\Delta}_{i j}^{\prime} \\
& \pm 2 \sum_{(i j)}^{s i k l i k} \tilde{J} \sin 2 \tilde{\Delta}_{i j}
\end{aligned}
$$

dengan $\operatorname{Re} \tilde{J}_{\alpha \beta}^{i j}$ adalah

$\operatorname{Re} \tilde{J}_{\alpha \beta}^{i j}=\frac{\left|\tilde{p}_{\alpha \beta}\right|^{2} \lambda_{i} \lambda_{j}+\left|\tilde{q}_{\alpha \beta}\right|^{2}+\operatorname{Re}\left(\tilde{p}_{\alpha \beta} \tilde{q}_{\alpha \beta}^{*}\right)\left(\lambda_{i}+\lambda_{j}\right)}{\tilde{\Delta}_{i j} \tilde{\Delta}_{12} \tilde{\Delta}_{23} \tilde{\Delta}_{31}}$

dan faktor Jarlskog dalam materi didapatkan

$$
\tilde{J}=\frac{\operatorname{Im}\left(\tilde{p}_{e \mu} \tilde{q}_{e \mu}^{*}\right)}{\tilde{\Delta}_{12} \tilde{\Delta}_{23} \tilde{\Delta}_{31}}
$$


Probabilitas osilasi neutrino baik transisi maupun survival dalam materi didapatkan dengan memasukkan bentuk eksplisit $\operatorname{Re} \tilde{J}_{\alpha \beta}^{i j}$ dan faktor Jarlskog dalam materi ke Pers.(47), didapatkan

$$
\begin{aligned}
P\left(\nu_{e} \rightarrow \nu_{\mu}\right) & =\tilde{A}_{e \mu} \cos \delta+\tilde{B} \sin \delta+\tilde{C}_{e \mu} \\
P\left(\nu_{e} \rightarrow \nu_{\tau}\right) & =-\tilde{A}_{e \mu} \cos \delta-\tilde{B} \sin \delta+\tilde{C}_{e \tau} \\
P\left(\nu_{\mu} \rightarrow \nu_{\tau}\right) & =\tilde{A}_{\mu \tau} \cos \delta+\tilde{B} \sin \delta+\tilde{C}_{\mu \tau}+\tilde{D} \cos 2 \delta \\
P\left(\nu_{e} \rightarrow \nu_{e}\right) & =\tilde{C}_{e e} \\
P\left(\nu_{\mu} \rightarrow \nu_{\mu}\right) & =\tilde{A}_{\mu \mu} \cos \delta+\tilde{C}_{\mu \mu}-\tilde{D} \cos 2 \delta \\
P\left(\nu_{\tau} \rightarrow \nu_{\tau}\right) & =\tilde{A}_{\tau \tau} \cos \delta+\tilde{C}_{\tau \tau}-\tilde{D} \cos 2 \delta
\end{aligned}
$$

dengan koefisien - koefisiennya secara eksplisit adalah

$$
\begin{aligned}
& \tilde{A}_{e \mu}=\sum_{(i j k)}^{\text {siklik }} \frac{-8\left[J_{r} \Delta_{21} \Delta_{31} \lambda_{k}\left(\lambda_{k}-\Delta_{31}\right)+\left(\tilde{A}_{e \mu}\right)_{k}\right]}{\tilde{\Delta}_{j k}^{2} \tilde{\Delta}_{k i}^{2}} \\
& \times \cos \tilde{\Delta}_{i j}^{\prime} \sin \tilde{\Delta}_{j k}^{\prime} \sin \tilde{\Delta}_{k i}^{\prime} \\
& \tilde{B}=\frac{8 J_{r} \Delta_{12} \Delta_{23} \Delta_{31}}{\tilde{\Delta}_{12} \tilde{\Delta}_{23} \tilde{\Delta}_{31}} \cos \tilde{\Delta}_{12}^{\prime} \sin \tilde{\Delta}_{23}^{\prime} \sin \tilde{\Delta}_{31}^{\prime} \\
& \tilde{C}_{e \mu}=\sum_{(i j)}^{s i k l i k} \frac{-4\left[\Delta_{31}^{2} s_{13}^{2} s_{23}^{2} c_{13}^{2} \lambda_{i} \lambda_{j}+\left(\tilde{C}_{e \mu}\right)_{i j}\right]}{\tilde{\Delta}_{i j} \tilde{\Delta}_{12} \tilde{\Delta}_{23} \tilde{\Delta}_{31}} \sin ^{2} \tilde{\Delta}_{i j}^{\prime} \\
& \tilde{C}_{e \tau}=\sum_{(i j)}^{s i k l i k} \frac{-4\left[\Delta_{31}^{2} s_{23}^{2} c_{23}^{2} c_{13}^{4}\left(\lambda_{i}-A\right)\left(\lambda_{j}-A\right)+\left(\tilde{C}_{\mu \tau}\right)_{i j}\right]}{\tilde{\Delta}_{i j} \tilde{\Delta}_{12} \tilde{\Delta}_{23} \tilde{\Delta}_{31}} \\
& \times \sin ^{2} \tilde{\Delta}_{i j}^{\prime} \\
& \tilde{A}_{\mu \tau}=\sum_{(i j k)}^{s i k l i k} \frac{-8\left[J_{r} \Delta_{21} \Delta_{31}\left(\lambda_{k}-A\right)\left(\lambda_{k}-A-\Delta_{31}\right)\left(c_{23}^{2}-s_{23}^{2}\right)+\left(\tilde{A}_{\mu \tau}\right)_{k}\right]}{\tilde{\Delta}_{j k}^{2} \tilde{\Delta}_{k i}^{2}} \\
& \times \cos \tilde{\Delta}_{i j}^{\prime} \sin \tilde{\Delta}_{j k}^{\prime} \sin \tilde{\Delta}_{k i}^{\prime} \\
& \tilde{C}_{\mu \tau}=\sum_{(i j)}^{s i k l i k} \frac{-4\left[\Delta_{31}^{2} s_{23}^{2} c_{23}^{2} c_{13}^{4}\left(\lambda_{i}-A\right)\left(\lambda_{j}-A\right)+\left(\tilde{C}_{\mu \tau}\right)_{i j}\right]}{\tilde{\Delta}_{i j} \tilde{\Delta}_{12} \tilde{\Delta}_{23} \tilde{\Delta}_{31}} \sin ^{2} \tilde{\Delta}_{i j}^{\prime} \\
& \tilde{D}=\sum_{(i j k)}^{s i k l i k} \frac{-8 \Delta_{21}^{2}\left(\lambda_{k}-A-\Delta_{31}\right)^{2} s_{12}^{2} c_{12}^{2} s_{23}^{2} c_{23}^{2} s_{13}^{2}}{\tilde{\Delta}_{j k}^{2} \tilde{\Delta}_{k i}^{2}} \\
& \times \cos \tilde{\Delta}_{i j}^{\prime} \sin \tilde{\Delta}_{j k}^{\prime} \sin \tilde{\Delta}_{k i}^{\prime} \\
& \tilde{C}_{e e}=1+\sum_{(i j)}^{\text {siklik }} \frac{4\left[\Delta_{31}^{2} s_{13}^{2} c_{13}^{2} \lambda_{i} \lambda_{j}+\left(\tilde{C}_{e e}\right)_{i j}\right]}{\tilde{\Delta}_{i j} \tilde{\Delta}_{12} \tilde{\Delta}_{23} \tilde{\Delta}_{31}} \sin ^{2} \tilde{\delta}_{i j}^{\prime}
\end{aligned}
$$




$$
\begin{aligned}
& \tilde{A}_{\mu \mu}=-\tilde{A}_{e \mu}-\tilde{A}_{\mu \tau} \\
& =-\sum_{(i j k)}^{\text {siklik }} \frac{-8\left[J_{r} \Delta_{21} \Delta_{31} \lambda_{k}\left(\lambda_{k}-\Delta_{31}\right)+\left(\tilde{A}_{e \mu}\right)_{k}\right]}{\tilde{\Delta}_{j k}^{2} \tilde{\Delta}_{k i}^{2}} \\
& \times \cos \tilde{\Delta}_{i j}^{\prime} \sin \tilde{\Delta}_{j k}^{\prime} \sin \tilde{\Delta}_{k i}^{\prime} \\
& -\sum_{(i j k)}^{s i k l i k} \frac{-8\left[J_{r} \Delta_{21} \Delta_{31}\left(\lambda_{k}-A\right)\left(\lambda_{k}-A-\Delta_{31}\right)\left(c_{23}^{2}-s_{23}^{2}\right)+\left(\tilde{A}_{\mu \tau}\right)_{k}\right]}{\tilde{\Delta}_{j k}^{2} \tilde{\Delta}_{k i}^{2}} \\
& \times \cos \tilde{\Delta}_{i j}^{\prime} \sin \tilde{\Delta}_{j k}^{\prime} \sin \tilde{\Delta}_{k i}^{\prime} \\
& \tilde{C}_{\mu \mu}=1-\tilde{C}_{e \mu}-\tilde{C}_{\mu \tau} \\
& =1-\sum_{(i j)}^{\text {siklik }} \frac{-4\left[\Delta_{31}^{2} s_{13}^{2} s_{23}^{2} c_{13}^{2} \lambda_{i} \lambda_{j}+\left(\tilde{C}_{e \mu}\right)_{i j}\right]}{\tilde{\Delta}_{i j} \tilde{\Delta}_{12} \tilde{\Delta}_{23} \tilde{\Delta}_{31}} \sin ^{2} \tilde{\Delta}_{i j}^{\prime} \\
& -\sum_{(i j)}^{\text {siklik }} \frac{-4\left[\Delta_{31}^{2} s_{23}^{2} c_{23}^{2} c_{13}^{4}\left(\lambda_{i}-A\right)\left(\lambda_{j}-A\right)+\left(\tilde{C}_{\mu \tau}\right)_{i j}\right]}{\tilde{\Delta}_{i j} \tilde{\Delta}_{12} \tilde{\Delta}_{23} \tilde{\Delta}_{31}} \sin ^{2} \tilde{\Delta}_{i j}^{\prime} \\
& \tilde{A}_{\tau \tau}=-\tilde{A}_{e \tau}-\tilde{A}_{\mu \tau} \\
& =-\sum_{(i j k)}^{\text {siklik }} \frac{8\left[J_{r} \Delta_{21} \Delta_{31} \lambda_{k}\left(\lambda_{k}-\Delta_{31}\right)+\left(\tilde{A}_{e \mu}\right)_{k}\right]}{\tilde{\Delta}_{j k}^{2} \tilde{\Delta}_{k i}^{2}} \\
& \times \cos \tilde{\Delta}_{i j}^{\prime} \sin \tilde{\Delta}_{j k}^{\prime} \sin \tilde{\Delta}_{k i}^{\prime} \\
& -\sum_{(i j k)}^{s i k l i k} \frac{-8\left[J_{r} \Delta_{21} \Delta_{31}\left(\lambda_{k}-A\right)\left(\lambda_{k}-A-\Delta_{31}\right)\left(c_{23}^{2}-s_{23}^{2}\right)+\left(\tilde{A}_{\mu \tau}\right)_{k}\right]}{\tilde{\Delta}_{j k}^{2} \tilde{\Delta}_{k i}^{2}} \\
& \times \cos \tilde{\Delta}_{i j}^{\prime} \sin \tilde{\Delta}_{j k}^{\prime} \sin \tilde{\Delta}_{k i}^{\prime} \\
& \tilde{C}_{\tau \tau}=1-\tilde{C}_{e \tau}-\tilde{C}_{\mu \tau} \\
& =1-\sum_{(i j)}^{s i k l i k} \frac{-4\left[\Delta_{31}^{2} s_{13}^{2} c_{23}^{2} c_{13} \lambda_{i} \lambda_{j}+\left(\tilde{C}_{e \tau}\right)_{i j}\right]}{\tilde{\Delta}_{i j} \tilde{\Delta}_{12} \tilde{\Delta}_{23} \tilde{\Delta}_{31}} \sin ^{2} \tilde{\Delta}_{i j}^{\prime} \\
& -\sum_{(i j)}^{s i k l i k} \frac{-4\left[\Delta_{31}^{2} s_{23}^{2} c_{23}^{2} c_{13}^{4}\left(\lambda_{i}-A\right)\left(\lambda_{j}-A\right)+\left(\tilde{C}_{\mu \tau}\right)_{i j}\right]}{\tilde{\Delta}_{i j} \tilde{\Delta}_{12} \tilde{\Delta}_{23} \tilde{\Delta}_{31}} \sin ^{2} \tilde{\Delta}_{i j}^{\prime}
\end{aligned}
$$

\section{SIMPULAN}

Perhitungan probabilitas osilasi neutrino telah dilakukan baik dalam vakum maupun materi. Diperoleh probabili- tas transisi dan survival neutrino dalam vakum adalah terlihat bahwa probabilitas neutrino dalam vakum maupun materi memiliki bentuk yang sama, hanya berbeda koefisienkoefisiennya saja.
[1] Super-Kamiokande Collaboration, Y. Fukuda et al., Phys.Rev. Lett., 81, 1562-1567 (1998); Phys.Lett., B433, 9-18 (1998); Phys.Rev.Lett., 82, 2644-2648 (1999).

[2] Z. Maki, M. Nakagawa, and S. Sakata, Prog.Theor.Phys., 28 870-
880 (1962),

[3] M. Freud, Phys.Rev., D64, 053003 (2001).

[4] J. Arafune, M. Koike, and J. Sato , Phys.Rev., D56, 3093-3099 (1997). 
[5] H. Minakata, and H. Nunokawa, Phys.Rev., D57, 4403-4417 (1998).

[6] C. Giunti, and C.W. Kim., Fundamentals of Neutrino Physics and Astrophysics (Cambridge University Press, London UK, 2007).

[7] M. Fukugita, and T. Yanagida, Physics of Neutrinos and Applications to Astrophysics (Springer-Verlag Berlin, Germany,
2003).

[8] S. Bilenky, Introduction to the Physics of Massive and Mixid Neutrinos (Springer-Verlag Berlin, Germany , 2010).

[9] K. Kimura, A. Takamura, and H. Yokomakura, Phys.Lett., B537, 86-94 (2002); Phys.Rev.D, D66, 073005 (2002); J.Phys., G29, 1839-1842 (2003). 